\section{Educación médica para la Atención Primaria de Salud: visión de los docentes y estudiantes}

\author{
MARIO PARADA-LEZCANO ${ }^{1}$, MARÍA INÉS ROMERO S. ${ }^{2}$, \\ FABIÁN MORAGA CORTÉS ${ }^{3, a}$
}

\section{Perceptions of faculty members and students about undergraduate training in primary health care}

Background: It is unknown if medical education is preparing physicians to successfully work at primary health care settings. Aim: To explore what are the perceptions of faculty members and students about the type of physician needed and if medical education is coherent with the practice of primary health care. Material and Methods: Fifteen semi-structured interviews to key informants from faculty members and ten focus groups with students were carried out. Results: Important influences of role modelling and hidden curriculum were found, especially in relation to the type of physician needed, generalist or specialist, and in relation to the places where the clinical practices were done. Although primary health care was declared in the profiles, most of clinical practices were done at hospitals and supervised by specialists. Working at primary health care is seen as a temporary work, not valued by professionals nor by the society. Conclusions: Medical Schools are not preparing professionals for primary health care but for hospital care and specialized medicine.

(Rev Med Chile 2016; 144: 1059-1066)

Key words: Medical Education, undergraduate; Primary Health Care; General Practice Physicians; Hidden Curriculum; Role Modeling; Teaching Methods.
'Departamento de Salud Pública, Escuela de Medicina, Universidad de Valparaíso. Región de Valparaíso, Chile.

${ }^{2}$ Instituto de Políticas Públicas en Salud, Universidad San Sebastián. Región Metropolitana, Chile. ${ }^{3}$ Universidad de Valparaíso. Valparaíso, Chile. asociólogo.

Fuente de apoyo financiero: Proyecto de investigación financiado por la Vicerrectoría de Investigación de la Universidad San Sebastián, No 2012-0006-R. La institución no tuvo injerencia en el diseño ni la ejecución del estudio.

Recibido el 2 de diciembre de 2015, aceptado el 23 de marzo de 2016.

Correspondencia a: Mario Parada Hontaneda 2653, Valparaíso, Región de Valparaíso. Teléfono: 56322603074 mario.parada@uv.cl
E 1 proceso de Reforma Sanitaria iniciado en Chile en 2005 plantea como su eje central la Atención Primaria de Salud (APS), considerando un modelo de salud familiar y redes asistenciales integradas ${ }^{1,2}$. Dicha centralidad no se ha concretado en el país ni en la mayoría de los países de América.

Estudios de la Organización Panamericana de la Salud (OPS) señalan que los problemas fundamentales de la Atención Primaria derivan de una elevada proporción de médicos especialistas versus generales-familiares y de una formación médica con énfasis biologicista; centrada en hospitales; ausencia de enfoque de promoción-prevención desde el inicio de la formación; carencia de for- mación integral desde perspectivas humanistas; falta de compromiso con la salud de la población lo que conduce a escasos conocimientos y habilidades para el manejo de la APS $^{3,4} y$, en Chile a una preparación insuficiente para dar respuesta a las necesidades generadas por la reforma sanitaria de 2005.

Dado lo anterior, OPS propone una formación basada en la comunidad, con orientación generalista, con contenidos de salud pública, salud familiar-comunitaria, enfoque de APS $^{3-7}$ e insta a los países miembros a implementar reformas en la educación médica que propicien sistemas de salud basados en la $\mathrm{APS}^{8}$, estableciendo como metas que el $80 \%$ de escuelas de ciencias de salud tengan 
orientación hacia APS y salud comunitaria; una fuerza laboral médica con una proporción mayor a 40\% de médicos en APS. Sin embargo, en 2013 el cumplimiento de los indicadores en la región fue $52,5 \%$ y $25,1 \%$ respectivamente ${ }^{9,10}$.

Un artículo reciente de los mismos autores del presente artículo ${ }^{11}$ analizó los perfiles de egreso publicados por las carreras de medicina de la Asociación de Facultades de Medicina de Chile (ASOFAMECH), constatando que ellos no están considerando cabalmente la política pública ni los objetivos sanitarios como ejes movilizadores de la formación. Se detectó falta de elementos como el pensamiento crítico y determinantes sociales de la salud, generándose dudas respecto a la articulación real del proceso formativo con la realidad socio-sanitaria nacional.

Otra evidencia que apoya estos antecedentes es el sostenido aumento de médicos especialistas en Chile $^{12-15}$. Explican este escenario factores como: precariedad de la carrera funcionaria en APS, bajas remuneraciones en relación al mercado privado, inadecuado perfil de egreso, centralidad de la enseñanza en el hospital, marcado acento en especialización e inequidades entre el sector público y privado ${ }^{16,17}$, todo lo cual implica obstáculos para incorporar las orientaciones de OPS en la formación médica.

No obstante, algunas escuelas han emprendido reformas curriculares que supuestamente orientan la formación en un sentido cercano al propuesto por OPS ${ }^{18-25}$. Sin embargo, los resultados de estos procesos no han sido documentados en profundidad.

La presente investigación tuvo como objetivo analizar la situación actual de la educación médica, específicamente en relación a la preparación para el ejercicio en APS, a través de la percepción de los actores respecto de cuál es el profesional que Chile necesita, el tipo de perfil generalista existente, dónde se realizan las actividades formativas prácticas y cómo se visualiza el ejercicio en APS.

\section{Material y Método}

Investigación cualitativa con enfoque fenomenológico. A partir de estudio previo de los mismos autores $^{11}$ se seleccionaron escuelas, públicas y privadas, dos con alta presencia de enfoque social y humanista en sus perfiles de egreso publicados, y dos con baja presencia. Entre septiembre de 2013 y mayo de 2014 se realizaron 15 entrevistas individuales semiestructuradas (E) a informantes claves (directivos, docentes no clínicos, docentes clínicos APS y clínicos hospitalarios), y 10 entrevistas grupales focales (EGF) a estudiantes de primer, cuarto y séptimo año.

A los participantes se les explicó el contenido y alcance de la investigación, asegurándoles anonimato y confidencialidad respecto de sus personas y de las instituciones que representaban. Una vez transcritas las entrevistas se utilizó Atlas Ti, empleando análisis de contenido tipo "marco" (frameworkanalysis), ciego, y se trianguló a través de investigadores.

\section{Resultados}

No se encontraron diferencias discursivas al comparar los tipos de escuelas estudiadas, públicas y privadas, por lo que los resultados se presentan considerando sólo las categorías de análisis (ver Tablas 1 y 2 para apoyar la lectura de los resultados).

\section{El médico que Chile necesita}

Los docentes señalan que el país requiere de médicos generales y especialistas a la vez. Se alude a la baja disponibilidad y mala distribución geográfica de estos últimos como un factor decisivo, reconociendo, sin embargo, que el foco de la política sanitaria respalda la formación de generalistas. Se constata el surgimiento de un discurso que apunta a formar un médico "integral".

También los docentes manifiestan que la formación de médicos generales está siendo reducida porque la población exige cada vez más ser atendida por especialistas.

\section{Formación generalista}

Aunque está estipulada en los perfiles de egreso, no se cumple cabalmente. Las razones aludidas de esta discordancia apuntan a la cultura médica trasmitida desde los docentes los estudiantes que dice que "es mejor ser especialista" y a los mecanismos de remuneración establecidos a lo largo de la trayectoria profesional donde los especialistas ganan más. Lo anterior se ve reforzado por las valoraciones profesionales que subestiman el rol de los médicos generales. Esto se hace patente en el 
Tabla 1. Citas textuales (verbatims) según categorías de análisis y actor relevante

\begin{tabular}{|c|c|}
\hline Categoría/Informante & Discurso (verbatim) \\
\hline \multicolumn{2}{|c|}{ El médico que Chile necesita } \\
\hline E2 Docente Clínico APS & $\begin{array}{l}\text { "...No son sólo médicos de Atención Primaria, sino que son médicos de Atención Primaria } \\
\text { que sean resolutivos, de manera de descomprimir los hospitales secundarios. Y especialistas } \\
\text { en regiones, y especialistas en todo el país en áreas críticas falentes; urgencias, oftalmología, } \\
\text { dermatología, otorrino" }\end{array}$ \\
\hline E7 Docente Directivo & $\begin{array}{l}\text { "Por otro lado la sociedad... cuando tú les preguntas qué es lo que quieren, la gente quiere } \\
\text { especialistas, no quiere médicos generales..." }\end{array}$ \\
\hline \multicolumn{2}{|l|}{ Formación generalista } \\
\hline E6 Docente Directivo & $\begin{array}{l}\text { "El currículum dice cosas que el interés de las personas desdice... finalmente la ambición de } \\
\text { los médicos o de la mayor parte de los médicos es tener alguna especialidad; la cultura, la } \\
\text { presión social, la forma de pago de los sistemas de salud, los incentivos... no están puestos } \\
\text { en el médico general, los incentivos están puestos en el médico especialista" }\end{array}$ \\
\hline E13 Docente Directivo & $\begin{array}{l}\text { "... Porque cuando uno es especialista tiene herramientas económicas distintas, por lo tanto, } \\
\text { tiene la posibilidad de escoger más dónde lo hace, cuándo lo hace, con quién lo hace..." }\end{array}$ \\
\hline EGF 6 Estudiante $4^{\circ}$ & $\begin{array}{l}\text { "Me gustaría desempeñarme... en el sistema público en atención terciaria, hospitales base, } \\
\text { porque personalmente a mí la APS no me gusta mucho. Me gusta tener más capacidad de } \\
\text { resolución" }\end{array}$ \\
\hline EGF 9 Estudiante $4^{\circ}$ & $\begin{array}{l}\text { "La visión general de la Universidad es que intenta formar médicos generales. Ahora, no sé } \\
\text { qué tanto se da esa situación, porque en realidad todas las clases las tenemos con médicos } \\
\text { especialistas, y no hay muchos espacios donde se fomente la visión de médico general. No sé } \\
\text { si se cumple mucho el objetivo" }\end{array}$ \\
\hline \multicolumn{2}{|l|}{ Actividades prácticas } \\
\hline E6 Docente No Clínico & $\begin{array}{l}\text { "En la práctica creo que la docencia sigue centrada en el médico tradicional, que es el médico } \\
\text { del Hospital... Por eso las expectativas de los estudiantes es el Hospital, es el hiper especialista, } \\
\text { y no el médico de atención primaria" }\end{array}$ \\
\hline EGF 4 Estudiante $7^{\circ}$ & $\begin{array}{l}\text { "El perfil que busca la universidad es un médico integral, que sea más que nada empático con } \\
\text { la gente, que pueda ayudarlos, y que se enfoque a la medicina general más que nada. Tratan } \\
\text { de formar médicos generales, bueno... eso es lo que dicen" }\end{array}$ \\
\hline \multicolumn{2}{|c|}{ Orientación hacia el trabajo en APS } \\
\hline EGF 9 Estudiante $4^{\circ}$ & $\begin{array}{l}\text { "Nosotros como estamos en cuarto, no hemos tenido trabajo tan in situ en la atención pri- } \\
\text { maria. Eso se ve en quinto y sobre todo en séptimo, que uno se va al internado rural, que } \\
\text { tienes por lo menos un mes metido ahí. Pero yo diría que la mayor parte del tiempo estamos } \\
\text { en hospital" }\end{array}$ \\
\hline EGF 4 Estudiante $7^{\circ}$ & $\begin{array}{l}\text { "La idea es salir orientado a lo que uno quiere, para no perder tiempo. Para salir con la mente } \\
\text { clara en lo que uno quiere; si va a postular a la APS primero, si va a postular a médico general } \\
\text { de zona, si vas a hacer al tiro la especialidad..." }\end{array}$ \\
\hline E2 Docente Clínico APS & $\begin{array}{l}\text { "O sea, faltan programas y docentes encargados de esto. Tú no puedes tampoco mandar a } \\
\text { atención primaria a un chiquillo, con un médico de APS que tiene } 10 \text { minutos para ver a un } \\
\text { paciente, es una deformación, no puedes. Tienes que tener a alguien con espacio y tiempo } \\
\text { adecuado" }\end{array}$ \\
\hline
\end{tabular}

reconocimiento de que las supuestas exigencias del trabajo médico actual son atenuadas por las mejores condiciones que ofrece el trabajo especialista.

En contraste, los estudiantes creen que las universidades imparten una formación generalista como un piso mínimo, con facilidades para posteriormente especializarse. Esto es visualizado positivamente, dado que conciben el ejercicio generalista como un estado transitorio, desde el cual pueden optar a la especialización, con un trabajo médico más tecnologizado y con mayores niveles de resolutividad. 
Tabla 2. Principales resultados según categorías y actor

\begin{tabular}{|c|c|c|}
\hline Categoría de análisis & Docentes & Estudiantes \\
\hline $\begin{array}{l}\text { El médico que Chile } \\
\text { necesita }\end{array}$ & $\begin{array}{l}\text { Se apela por la necesidad de formar gene- } \\
\text { ralistas y especialistas paralelamente, para } \\
\text { conciliar el foco de la política pública con las } \\
\text { preferencias de las personas }\end{array}$ & Sin discurso* \\
\hline Formación generalista & $\begin{array}{l}\text { Se reconoce como una declaración institucio- } \\
\text { nal no cumplida a cabalidad por los incentivos } \\
\text { del proceso formativo y de la carrera profesio- } \\
\text { nal de los médicos. Ambos condicionan la per- } \\
\text { cepción de los generalistas como profesionales } \\
\text { de menor categoría }\end{array}$ & $\begin{array}{l}\text { Se concibe como los conocimientos básicos } \\
\text { necesarios para posteriormente optar a la } \\
\text { especialización. Se reconoce el impacto de } \\
\text { los docentes, que potencian el interés en } \\
\text { especializarse }\end{array}$ \\
\hline Actividades prácticas & $\begin{array}{l}\text { Se explicita que éstas se desarrollan mayor- } \\
\text { mente en hospitales. No hay cuestionamiento } \\
\text { a esta situación, se asume como una realidad } \\
\text { no susceptible de modificarse }\end{array}$ & $\begin{array}{l}\text { Existe un discurso crítico que cuestiona la su- } \\
\text { premacía de las actividades hospitalarias, por } \\
\text { el temor a no desenvolverse con propiedad en } \\
\text { el nivel primario durante los primeros años de } \\
\text { ejercicio laboral }\end{array}$ \\
\hline $\begin{array}{l}\text { Orientación hacia el } \\
\text { trabajo en APS }\end{array}$ & $\begin{array}{l}\text { Se describen escasos espacios para la labor pe- } \\
\text { dagógica en la APS, a partir de los obstáculos } \\
\text { para establecer convenios docentes-asisten- } \\
\text { ciales, a la carga laboral de este nivel y a la } \\
\text { disponibilidad de docentes }\end{array}$ & $\begin{array}{l}\text { Se piensa como un lugar de trabajo transito- } \\
\text { rio, desde el cual poder optar a la formación } \\
\text { especializada en los hospitales }\end{array}$ \\
\hline
\end{tabular}

*Esta categoría de análisis no fue considerada en el guion de las entrevistas grupales focales aplicadas a estudiantes, dado que se consideró que los docentes tenían mayor preparación para responder el tema.

Sin embargo, se observó un discurso de estudiantes que reconoce el impacto que tiene el rol de los docentes en sus preferencias y se constata que las declaraciones institucionales "generalistas" no están en sintonía con el tipo de docentes, que son mayoritariamente especialistas.

\section{Actividades prácticas}

Tanto docentes como estudiantes plantean que las actividades prácticas se desarrollan mayoritariamente en hospitales. Los docentes afirman que los estudiantes se encuentran satisfechos con esta situación.

No obstante, los estudiantes tienen un discurso crítico invisibilizado, que no aprueba el énfasis que se da a los contenidos vinculados a las especialidades hospitalarias, pues se intuye que al despriorizar la APS existirán falencias en el desarrollo profesional durante los primeros años de ejercicio.

\section{Orientación hacia el trabajo en APS}

Las escuelas estudiadas tienen internados desarrollados en APS y prácticas en distintos ramos que son esporádicas y/o voluntarias. Además, se señala que el contacto con la APS es extremadamente tardío.

La APS como lugar de trabajo médico se vislumbra como transitorio, siendo lo buscado la especialización, aun desconociendo la disciplina particular a elegir. Algunos docentes aducen que uno de los factores que incide en la falta de esta orientación es la baja disponibilidad de docentes con espacios adecuados para el ejercicio pedagógico, para poder ir más allá de la labor asistencial.

\section{Discusión}

Las citas textuales de los discursos de los participantes que sintetizan ideas centrales se muestran en la Tabla 3.

La percepción de que se requieren médicos generales y especialistas a la vez, es coherente con los antecedentes de la realidad nacional ${ }^{10-13}$. Esta situación tiene como trasfondo la arraigada concepción de que el ejercicio generalista sólo se dará de manera transitoria y/o circunstancial, hecho que se constataba en la década de los 60 en el programa de Médicos Generales de Zona y que 
Tabla 3. Citas textuales (Verbatims) de discursos según actor relevante respecto del ejercicio en la atención primaria de salud

\begin{tabular}{|c|c|}
\hline Informante & Discurso (Verbatim) \\
\hline FG 4 Estudiante $7^{\circ}$ & $\begin{array}{l}\text { "Nos dicen (los tutores) que en los consultorios van a trabajar los más malos, los que no se la } \\
\text { pueden" }\end{array}$ \\
\hline E2 Académico clínico APS & $\begin{array}{l}\text { "Chile necesita un médico integral. Que tenga un enfoque biopsicosocial claramente. Necesi- } \\
\text { tamos gente que junte la ciencia con la empatía y con la sensibilidad social. Yo lo veo más que } \\
\text { un investigador o un especialista... es un médico que tenga una sólida formación científica, } \\
\text { que tenga una sólida formación en medicina basada en la evidencia..." }\end{array}$ \\
\hline E6 Directivo & $\begin{array}{l}\text { "La política no lo ha podido resolver porque de algún modo la sociedad se ha ido moviendo } \\
\text { hacia otro plano, finalmente la gente lo que quiere consumir hoy son especialistas, no médicos } \\
\text { generales" }\end{array}$ \\
\hline FG 4 Estudiante $7^{\circ}$ & $\begin{array}{l}\text { "Yo creo que por la misma influencia de los especialistas, o sea la mayoría de los profesores son } \\
\text { especialistas, y de alguna u otra manera influyen en la decisión que uno toma, o en las expec- } \\
\text { tativas que uno tiene de la carrera... entonces la formación, o sea la malla de estudio involucra } \\
\text { mucha especialidad. Y compartes mucho más con especialista que con médicos generales" }\end{array}$ \\
\hline
\end{tabular}

se mantiene en la actualidad con los programas destinados a médicos de APS que tienen como incentivo explícito la futura especialización.

Es interesante que los docentes usen un discurso que homologa el concepto de "médico integral" al de "médico general". En esta conceptualización está implícita la idea de formar un profesional "indiferenciado", con la potencialidad intrínseca para desempeñarse en cualquier nivel de atención, complejidad o ámbito de la medicina, algo así como "stem cell". No obstante, esta idea no es congruente con la formación generalista recomendada por OPS, que propicia un médico preparado para trabajar en APS y no en cualquier otro espacio asistencial.

También es muy intenso el discurso de la falta de especialistas y del requerimiento explícito de este tipo de médicos por parte de la mayoría de la población. Este discursos está sostenido en el modelo biomédico, hospitalocéntrico y mercantilizado de la atención médica chilena en cuya industria de aseguramiento y prestación privada no existe la APS y la gente "elige libremente" al especialista que quiere. Excepcionalmente los planes privados incluyen médicos de familia o redes cerradas que impliquen paso por un nivel semejante a la APS pública.

Los docentes tienen la percepción que los estudiantes valoran positivamente que la formación tenga una tendencia hacia la especialización futura. Sin embargo, se observó un discurso estudiantil crítico, que visualiza distanciamiento entre contenidos entregados y futuro ejercicio profesional, generando grados de incertidumbre desconocidos y/o menospreciados por los docentes.

El reconocimiento de las escasas instancias para la formación en APS y la realización de la mayoría de las prácticas en hospitales complejos, es potente y se asume como una realidad objetiva y difícil de modificar; un hecho ante el cual habría que rendirse, a pesar de percibir que va en contra de lo adecuado, lo deseado, lo recomendado.

Las inconsistencias entre las declaraciones institucionales (perfiles) y los procesos formativos dan cuenta del impacto que representan el currículo informal y el currículum oculto en la educación médica del país. Lo que se aprende en las escuelas no es sólo lo que dicen los programas ni lo que se enseña en el aula, el laboratorio o el campo clínico. Hafferty $^{26}$ en 1998 describía tres componentes interrelacionados: el currículo formal, el currículo informal y el currículo oculto, destacando que, de alguna manera, el currículo informal y el oculto funcionaban en contraste con el formal.

El formal es público, conocido y se desarrolla en tiempos y espacios oficiales. El informal no es conocido, se desarrolla en cualquier lugar en que interactúen docentes y estudiantes y se asocia y explica la importancia del modelaje en la educación médica. El currículo oculto corresponde a un conjunto de influencias que funcionan a nivel de la estructura y cultura organizacional con sus 
costumbres y rituales. Pueden tener una influencia negativa en la formación, incluso actuar de modo opuesto los programas oficiales.

Diversos autores que destacan las influencias de currículo informal y oculto ${ }^{27-32}$ concluyen que se requiere un reconocimiento y tomar conciencia de su existencia, lo cual puede transformarlos en una herramienta para revisar y humanizar la educación médica clínica.

Los actores del proceso educativo mantienen prácticas profundamente arraigadas en la cultura institucional, demostrando resistencia a los cambios de paradigmas, pese a que formalmente hay una adhesión a los objetivos explicitados ${ }^{33}$, lo que explica el distanciamiento entre los perfiles de egreso y el relato manifestado por los actores.

Asimismo, en el ámbito del currículo informal en la formación médica, se ha demostrado, que el modelaje de los docentes ${ }^{34-36}$ propicia la reproducción del modelo profesional hospitalocéntrico, curativo y biologicista, incentivando estos rasgos en las preferencias del estudiantado, en oposición a una educación basada en la comunidad, con orientación generalista, con contenidos de salud pública, y salud familiar-comunitaria, en definitiva con enfoque de APS.

La tendencia actual de una enseñanza médica hecha por especialistas, afecta negativamente las decisiones para optar por enfoques generalistas del ejercicio profesional. Ello se había mostrado ya en $1996^{37}$ cuando $17 \%$ de los egresados había cambiado su elección de forma de ejercicio profesional debido a los comentarios negativos y opiniones estereotipadas de sus docentes ("bad mouthing") afectando especialmente la elección del ejercicio de la cirugía, la medicina familiar y la medicina interna. Esto reafirma la importancia de reconocer estas influencias, buscar de dónde proceden y enfrentarlas.

Los resultados obtenidos están en sintonía con los hallazgos de un estudio realizado en una de las facultades de ASOFAMECH, en donde se documentó una considerable influencia del modelo médico trasmitido hacia los estudiantes desde los docentes, los que son descritos con poco compromiso con los pacientes, excesivamente mercantilistas y con predominancia de los intereses individuales por sobre los generales ${ }^{38}$.

En conclusión, las escuelas investigadas no están formando profesionales para el ejercicio preferente en APS, se conforma un escenario ad- verso para la incorporación de las orientaciones de OPS: creencia que el país requiere de médicos especialistas y generales en igual importancia; inconsistencias para llevar a la práctica el enfoque generalista establecido en los perfiles de egreso; escasos espacios para la formación para la APS; reproducción cultural hegemónica, especialmente a través del currículo informal y oculto.

La evidencia encontrada es preocupante, pues está documentado que los Sistemas de Salud basados en la APS logran mejores indicadores socio-sanitarios, mayores grados de eficiencia, y contienen el aumento de costos de la atención médica $^{39}$.

Se sugiere la formulación de una política pública de carácter nacional y fortalecer la regulación de la formación médica, para hacer congruente la respuesta a las necesidades de salud-enfermedad-atención de la población con los procesos formativos.

\section{Referencias}

1. Molina C. El médico para Chile en el siglo XXI. Cuad Med Soc 1995; 36 (Extraordinario): 37-42.

2. Bass C. ¿Cuál es el médico que necesita nuestro país? [Sitio en internet]. El quinto poder. Hallado en: http:// www.elquintopoder.cl/salud/cual-es-el-medico-quenecesita-nuestro-pais/ (Acceso el 20 de mayo de 2015).

3. OPS. Educación Médica hacia la APS renovada y libros de texto. Informe de la reunión de Buenos Aires, Argentina. Rosa Borrel y Rodolfo Kauffman (relatores). [Sitio en internet]. Portal web de la Organización Panamericana de la Salud. Hallado en: http://www.observatoriorh.org/?q=node/523 (Acceso el 2 de septiembre de 2013).

4. Godue C, Borrel R. Ideas principales de la conferencia de Beyond Flexner: Misión social de la Educación Médica. Tulsa, Oklahoma. [Sitio en internet] Observatorio Regional de Recursos Humanos en Salud. Hallado en: http://www.observatoriorh.org/?q=node/391 (Acceso el 2 de septiembre de 2013).

5. Godue C, Borrell R. La Acreditación de Programas de Formación Médica y la Orientación hacia la APS. Serie $\mathrm{N}^{\circ} 3$ La Renovación de la Atención Primaria en las Américas. [Sitio en internet]. Observatorio Regional de Recursos Humanos en Salud. Hallado en: http://www. paho.org/hq/index.php?option=com_content\&view=category\&layout $=$ blog\&id $=3176 \&$ Itemid $=3536 \&$ lang $=e s$ (Acceso el 3 de septiembre de 2013). 
6. Borrell R. Relato de un diálogo virtual exitoso en el portal del Observatorio de Recursos Humanos de Salud de la OPS/OMS. [Sitio en internet]. Observatorio Regional de Recursos Humanos en Salud. Hallado en: www. observatoriorh.org (Acceso el 3 de septiembre de 2013).

7. OPS. La formación en medicina orientada hacia la atención primaria de salud. Serie La renovación de la Atención Primaria de Salud en las Américas. [Sitio en internet]. Portal web de la Organización Panamericana de la Salud. Hallado en: http://new.paho.org/hq/dmdocuments/ 2010/APS-Formacion_Medicina_Orientada (Acceso el 2 de agosto de 2013).

8. OPS. Resolución CD52.R13. Recursos humanos para la salud: aumentar el acceso al personal sanitario capacitado en Sistemas de Salud basados en la Atención Primaria de Salud. 52 Consejo Directivo - 65ª Sesión del Comité Regional. Washington, 2013.

9. Observatorio Regional de Recursos Humanos en Salud. Medición y Monitoreo de Metas Regionales en Recursos Humanos en Salud. OPS, 2015. Hallado en: http://www. observatoriorh.org/?q=node/368 (Acceso el 20 de mayo de 2015).

10. OPS. Indicadores básicos 2014. 20 años. Situación de Salud de las Américas. OPS-OMS. Washington. 2014.

11. Parada M, Romero M, Moraga F. Perfiles de egreso de las carreras de Medicina en Chile. Rev Med Chile 2015; 143 (4): 512-9.

12. Bastías S, Marshall R, Zufina P. Número de médicos en Chile: estimaciones, proyecciones y comparación internacional. Rev Med Chile 2000; 128 (10): 1167-76.

13. Saavedra F. Proyección de la disponibilidad de profesionales de la salud en Chile al 2020. Cuad Med Soc 2010; 50 (4): 307-14.

14. Montero J, Muñoz F, Rosselot E, Valdivieso V, Barba R. La medicina general en la organización médica actual. Rev Med Chile 1996; 124 (8): 1006-14.

15. Román $\mathrm{O}$, Acuña $M$, Señoret M. Disponibilidad de médicos en Chile al año 2004. Rev Med Chile 2006; 134 (8): 1057-64.

16. Breinbauer H, Fromm G, Fleck D, Araya L. Tendencia en el estudiante de medicina a ejercer como médico especialista. Rev Med Chile 2009; 137 (7): 865-72.

17. Román $\mathrm{O}$, Pineda $\mathrm{S}$, Señoret M. Perfil y número de médicos generales que requiere el país. Rev Med Chile 2007; 135 (9): 1209-15.

18. Oyarzún F. Perspectiva antropológica en la formación médica: experiencia de la Universidad Austral de Chile. Rev Med Chile 1994; 122 (12): 1428-9.

19. Goic A. Un nuevo currículo para la Escuela de Medicina de la Universidad de Chile. Cuad Med Soc 1995; 36 (número extraordinario): 34-6.
20. Rosselot E. Reorganización en la educación médica. Aspectos esenciales de la reestructuración de la Facultad de Medicina de la Universidad de Chile. Rev Med Chile 1997; 125 (7): 783-91.

21. Goic A, Florenzano R, Velasco C. Análisis de la formación humanística y psicosocial en el pre-grado de la carrera de medicina. Rev Med Chile 1985; 113 (5): 453-62.

22. Facultad de Ciencias Médicas de la Universidad de Santiago de Chile. El médico formado en la USACH. Rev Clínica y Ciencia 2002; 1 (5): 49-57.

23. Parada M, McColl P, Barros M, González R. Una estrategia integral para mejorar la educación médica en la Universidad de Valparaíso. Universidad de Valparaíso; 2007.

24. Goic A. Seminario sobre formación de médicos en la actualidad en Chile. Rev Med Chile 2003; 131 (2): 20912.

25. Hebel E. Presentación de la Universidad de la Frontera. [Sitio en internet]. Observatorio Regional de Recursos Humanos en Salud. Hallado en: www.observatoriorh. org (Acceso el 5 de septiembre de 2013).

26. Hafferty F. Beyond Curriculum Reform: Confronting Medicine's Hidden Curriculum. Academic Medicine 1998, 73 (4): 403-7.

27. Michalec B. The pursuit of medical knowledge and the potential consequences of the hidden curriculum. Health 2011; 16 (3) 267-81.

28. Rojas A. Currículum oculto en medicina: una reflexión docente. Rev Med Chile 2012; 140 (9): 1213-7.

29. Lempp H, Seale C. The hidden curriculum in undergraduate medical education: qualitative study of medical students' perceptions of teaching. BMJ 2004; 329 (2): 770-3.

30. Chuang A, Nuthalapaty F, Casey P, Kaczmarczyk J, Cullimore E, Dalrymple J, et al. To the point: reviews in medical education. Taking control of the hidden curriculum. American Journal of Obstetrics and Gynecology 2010; 203 (4): 316-22.

31. Gaufberg E, BataldenM, Sands R, Bell S. The Hidden Curriculum: What Can We Learn From Third-Year Medical Student Narrative Reflections? Academic Medicine 2010; 85 (11): 1709-16.

32. Murakami M, Kawabata H, Maezawa M. The perception of the hidden curriculum on medical education: an exploratory study. Asia Pacific Family Medicine 2009; 8 (1): 9 .

33. Margolis E, Soldatenko M, Acker S, Gair M. Peekaboo. Hiding and outing the curriculum. In Eric Margolis (ed.) The Hidden curriculum in higher education. ROUTLEDGE. New York and London. 2001. 
34. Cruess S, Cruess R, Steinert Y. Role modeling-making the most of a powerful teaching strategy. BMJ 2008; 336 (7646): 718-21.

35. Kenny N, Mann K, MacLeod H. Role modeling in physicians' professional formation: reconsidering an essential but untapped educational strategy. Acad Med 2003; 78 (12): 1203-10.

36. Margolis E, Romero M. "In the image and likeness..." How mentoring functions in the hidden curriculum. En: Margolis E (ed.), The Hidden curriculum in higher education. New York and London: ROUTLEDGE; 2001. p 79-97.

37. Hunt D, Scott C, Zhong S, Goldstein E. Frequency and Effect of Negative Comments ("Badmouthing") on
Medical Students' Career Choices. Academic Medicine 1996; 71 (6): 665-9.

38. Orellana C, Rojas M, Silva M. Influencia del modelo e imagen del médico durante la formación de pregrado en un grupo de estudiantes de medicina de la Universidad de Antofagasta. Acta Bioethica 2010; 16 (2): 198-206.

39. OPS. Redes integradas de Servicios de Salud. Conceptos, opciones de Política y Hoja de Ruta para su implementación en las Américas. Serie la renovación de la Atención Primaria de Salud en las Américas. [Sitio en internet]. Portal web de la Organización Panamericana de la Salud. Hallado http://www.paho.org/uru/ index.php?option=com_docman\&task=doc_view\&gi$\mathrm{d}=145$ \&Itemid=250 (Acceso el 05 de octubre de 2015). 